Cahiers $d u$ MONDE RUSSE

\section{Cahiers du monde russe}

Russie - Empire russe - Union soviétique et États indépendants

$50 / 4 \mid 2009$

Varia

\title{
Peter Gatrell, Nick Baron, eds., Warlands
}

\section{Catherine Gousseff}

\section{OpenEdition \\ Journals}

Édition électronique

URL : https://journals.openedition.org/monderusse/7230

DOI : $10.4000 /$ monderusse. 7230

ISSN : $1777-5388$

\section{Éditeur}

Éditions de l'EHESS

\section{Édition imprimée}

Date de publication : 15 décembre 2009

Pagination : 905-909

ISBN : 978-2-7132-2261-0

ISSN : $1252-6576$

\section{Référence électronique}

Catherine Gousseff, "Peter Gatrell, Nick Baron, eds., Warlands », Cahiers du monde russe [En ligne], 50/4 | 2009, mis en ligne le 12 janvier 2011, consulté le 03 septembre 2022. URL : http:// journals.openedition.org/monderusse/7230; DOI : https://doi.org/10.4000/monderusse.7230

Ce document a été généré automatiquement le 3 septembre 2022

Tous droits réservés 


\title{
Peter Gatrell, Nick Baron, eds., Warlands
}

\author{
Catherine Gousseff
}

\section{RÉFÉRENCE}

Peter GATRELl, Nick BARON, eds., Population Ressetlement and State Reconstruction in the Soviet-East European Borderlands, 1945-1950. Warlands. New York : Palgrave Macmillan, 2009, 276 p.

1 Depuis les années 1990, à la faveur du renouveau historiographique sur la genèse de l'après-guerre en Europe centrale et de l'actualité des pratiques d'épuration ethnique liées aux guerres de l'ex-Yougoslavie, les travaux se sont multipliés sur la grande remue d'hommes qui a accompagné l'établissement du nouvel ordre européen à partir de 1945. Warlands se présente dans la continuité de publications collectives visant, à travers différentes études de cas, à dresser un panorama de la complexité des migrations de la période de sortie de guerre. Mais la spécificité de Warlands est de s'attacher, comme le sous-titre de l'ouvrage l'indique, aux territoires les plus à l'est de l'Europe, bordant l'URSS ou en URSS même, qui ont été relativement peu étudiés.

2 L'ouvrage s'ouvre sur une dense introduction de Peter Gatrell, maître d'œuvre, avec Nick Baron, de cette publication qui met en perspective les attendus de l'entreprise, tant dans l'espace étudié - une Europe, à l'Est, plusieurs fois remodelée politiquement au cours du $\mathrm{xx}^{\mathrm{e}}$ siècle et qui a expérimenté, à des degrés variables, les deux occupations nazie et soviétique - que dans les articulations entre pratiques de pouvoir à l'égard des migrants et expérience humaine du déplacement. Ces approches ambitieuses sont appliquées à différents types de situations, dont Peter Gatrell privilégie, dans sa réflexion, celle des Displaced Persons (DPs) autour, en particulier, de la figure du camp. On ne s'étonnera pas de cette attraction, compte tenu, notamment, de la richesse du questionnement que pose le cas spécifique de l'internement des déplacés de la guerre au cœur de l'Europe administrée par les Alliés. La multiplicité des acteurs impliqués (des responsables 
politiques au personnel d'encadrement des camps, en passant par les multiples bénévoles d'organisations caritatives), la diversité des groupes de déplacés, qu'il s'agisse de leurs nationalités, de l'origine, contrainte ou volontaire, de leur présence en Allemagne, la nature de l'internement, entre assistance et contrôle, font de l'histoire des DPs un laboratoire d'investigations très diverses. Ce laboratoire permet tout d'abord d'interroger l'appréhension faite des identités nationales dans une période où l'appartenance ethnique constitue l'un des facteurs décisifs des politiques de migrations organisées (notamment dans le cas des rapatriements forcés), où elle apparaît chez les migrants comme une ressource majeure de refondation individuelle et de solidarité collective, où elle se trouve confrontée à un encadrement transnational. De ce point de vue, l'introduction de Peter Gatrell met surtout l'accent sur la première partie de l'ouvrage, découpé selon les trois situations majeures rencontrées dans l'immédiate après-guerre. Intitulée « Transit », la première partie regroupe des contributions sur les camps de DPs, abordés par le biais de certaines communautés nationales et d'une organisation privée d'assistance. Le second volet, "Retours", se concentre sur les rapatriements vers l'URSS, la perception des nouveaux arrivants, la politique soviétique d'incitation à l'immigration. La troisième section, « Traverser les frontières », est dédiée aux transferts de populations qui ont accompagné le déplacement du territoire polonais vers l'ouest. Une dernière partie conclusive est consacrée, elle, aux politiques de la mémoire.

3 Sans remettre en cause la cohésion de l'ouvrage, sa lecture suggère d'autres correspondances qui soulignent d'une manière différente les points forts de cette publication. Un premier grand thème se dégage ainsi autour des " peuples de l'entredeux »; ceux-ci, qu'ils soient baltes ou ukrainiens, ont connu les deux occupations nazie et soviétique, et, par leur présence en Allemagne à la fin de la guerre, se trouvent sous le feu de la suspicion de collaboration. Or cette suspicion, avec ce qu'elle a entraîné de contrôles et d'interrogatoires personnels, est généralisée. Longtemps, les rapatriements massifs d'Ostarbeiter et d'autres déplacés forcés vers l'URSS ont été appréhendés sous un angle exclusivement répressif. Nick Baron, dans sa rétrospective des formes de filtration ou contrôles des rapatriés en URSS, nuance cette représentation en faisant davantage valoir ce que la procédure de filtration avait de fonctionnel dans l'inculcation, aux nouveaux entrants, des normes éthiques et politiques de l'Union soviétique. Après d'importants travaux d'historiens (en particulier celui de Jurij Arzamaskin ${ }^{1}$ ) qui ont conduit à relativiser l'importance des répressions, sous forme de déportations, des rapatriés d'Allemagne, N. Baron montre, lui, à travers le déroulement des procédures de contrôle, comment les interrogatoires, en quoi la perception soviétique du condamnable ou du louable, reformulaient les normes de la loyauté soviétique face aux expatriés. Cette lecture de la procédure de filtration comme phase initiatique d'une réintégration sociale ne nie pas la posture durable de fragilité dans laquelle restent les anciens expatriés, mais elle tend néanmoins à nuancer l'exceptionnalité soviétique en matière de contrôle.

Ce constat se dégage d'autant plus nettement que les contributions portant sur les camps de DPs à l'Ouest, insistent, quant à elles, sur le caractère traumatique des interrogatoires et autres contrôles régulièrement effectués auprès des réfugiés, sur le sentiment profond d'insécurité qui en a résulté dans les différents groupes nationaux et comment cela a influencé leur repli sur soi. L'empreinte du screening apparaît avec insistance dans l'analyse que fait Thomas Balkelis du camp comme "institution totale», en prenant à témoin le cas des DPs lituaniens. Par une autre approche, celle des organisations 
politiques des DPs lettons, Aldis Purs retrace la façon dont ces porte-parole ont été amenés à reconfigurer leur discours et leur passé face aux pressions conjointes des contrôles dans les camps et des nouvelles normes véhiculées par les Alliés. Ainsi, malgré la diversité des trajectoires, de rapatriement vers l'URSS ou de nouveau départ outreAtlantique, les DPs ont en commun d'avoir été soumis à une procédure de vérification politique de leur passé et, plus largement, d'avoir subi le poids de la suspicion, à l'Ouest comme à l'Est.

5 Un autre thème fort de ce volume concerne la politique soviétique de repeuplement de l'URSS, qui est donnée à voir ici à travers différents cas de figure, du plus pacifique au plus répressif. La campagne d'incitation au retour des diasporas arméniennes (principalement du Proche-Orient), analysée par Joanne Laycock dans une brillante synthèse, présente un aspect mal connu de l'après-guerre. Il s'agit de cette politique, certes circonscrite, d'immigration mise en place par les dirigeants soviétiques pour pallier le déficit démographique ainsi que la pénurie de compétences, et qui a connu un succès mitigé. Le terme d'incitation est, lui, peu adéquat pour caractériser les transferts de populations qui ont résulté $\mathrm{du}$ redécoupage des territoires frontaliers polonosoviétiques et conduit au déplacement de près de 500000 Ukrainiens de Pologne vers l'URSS. Dans sa rétrospective, Kateryna Stadnik souligne essentiellement le poids des contraintes matérielles, pressions et violences subies au cours de ces transferts forcés. Par rapport à ceux-ci se dégage néanmoins un cas plus extrême qui est celui des déportations massives vers l'URSS, orchestrées par les Soviétiques à l'égard des habitants de Haute-Silésie assimilés à des Allemands. Ce pan d'histoire, que retrace Ewa Ochman, est encore mal exploré, au point que l'estimation du nombre de déportés oscille toujours entre 30000 et 90000 personnes. Mais il rend compte de la véritable rafle d'hommes opérée dans les territoires libérés d'Europe centrale, qui a conduit des centaines de milliers d'étrangers vers les grands chantiers de production et de reconstruction de l'Union soviétique. Au-delà de ces schémas d'importation des hommes, la question du repeuplement de l'URSS est abordée à travers le cas de Leningrad, plus précisément par le biais du comportement des Leningradois face aux nouveaux arrivants, perçus au lendemain de la guerre comme une menace, notamment sur le plan sanitaire. La contribution de Siobhan Peeling se concentre sur l'appréhension de ces migrants dans un contexte de fort dérèglement des conditions d'hygiène urbaines, mais au détriment, regrettable, de toute tentative visant à identifier cette nouvelle population et à cibler son impact dans le repeuplement de la ville.

Bien que l'appréhension de l'identité nationale soit présente, en filigrane, quasiment dans tous les chapitres de l'ouvrage, celle-ci est frontalement abordée à travers quelques contributions spécifiques. Ainsi, dans une rétrospective sur la politique polonaise conduite à l'égard des minorités de l'après-guerre, Konrad Zielinski retrace la radicalité des mesures prises, en particulier à l'égard des Allemands, dans le processus de construction d'un État mono-ethnique où seuls, et jusque vers 1948-1949 seulement, les juifs semblent relativement épargnés par la volonté d'uniformisation socioculturelle prônée par le nouveau régime. Dans l'après-guerre, plus rares ont été les voix se revendiquant d'une tradition internationale et œuvrant dans ce sens. L'analyse originale que développe Jenny Carson à propos de l'intervention des Quakers dans les camps de DPs offre de ce point de vue un contrepoint intéressant. Ceux-ci cherchèrent en effet à désenclaver les groupes nationaux en encourageant une stratégie d'incitation à l'emploi, en développant une politique culturelle ad hoc, etc. Mais ils furent néanmoins conduits à agencer leurs idéaux à ceux des communautés, et à admettre, par exemple, à travers les 
manifestations à caractère folklorique qu'ils soutinrent, l'importance de l'appartenance nationale comme identité-ressource dans le dénuement de l'exil. Et ces Quakers apparaissent, à travers les documents cités, comme des témoins de l'intériorisation, chez les DPs, des haines nationales héritées de la guerre.

7 Warlands offre ainsi un foisonnement de réflexions et de points de vue très stimulants sur les migrations de sortie de guerre, même si la qualité des contributions se révèle très inégale, l'ouvrage mêlant des sujets très novateurs, documentés par d'importantes sources d'archives, à des synthèses, plus attendues, basées sur une littérature classique, voire secondaire. De même, certains aspects forts du questionnement sur la période ont été délaissés. En considérant exclusivement les migrations de la population civile, la majorité des auteurs ont exclu de leur analyse le facteur militaire pourtant si présent (qu'il s'agisse de l'encadrement, de la configuration des pratiques de cantonnement et d'organisation des migrations, etc.). On peut regretter encore que la polarisation sur l'expérience du camp n'ait pas été plus précisément resituée dans le grand défrichement en cours de l'histoire des DPs qui met en évidence la part très importante des réfugiés hors des camps, qui s'attelle à reconstituer l'univers composite des déracinés de la guerre auquel se mêlent les minorités allemandes expulsées d'Europe centrale. Cependant, audelà des inévitables lacunes, Warlands se distingue en particulier par le va-et-vient continu des approches. En effet, les chapitres ont généralement comme point commun de faire une large place, dans l'analyse, aux voix des témoins, aux récits des parcours, fournissant un aperçu de la richesse des témoignages disponibles, mais peu connus en raison des contraintes linguistiques. L'une des cohésions fortes de l'ouvrage réside ainsi dans cette tentative remarquable d'articuler la grande histoire des migrations, où il faut raisonner en millions d'hommes, à celle de l'humain migrateur, toujours singulier dans sa façon de traverser la vie et les épreuves qu'elle donne à subir.

\section{NOTES}

1. Jurij Azramaskin, Založniki vtoroj mirovoj voiny : repatriacija Sovetskih graždan v 1944-1953 gg [Les otages de la Seconde Guerre mondiale: le rapatriement des citoyens soviétiques, 1944-1953], M.: Stanitsa, 2001. 